\title{
Analysis of orthogonal signal of dual-mass micro-machined gyroscope
}

\author{
Zhanghui Wang ${ }^{1, *}$,Fei $\mathrm{Qi}^{2}$, Anping Qiu ${ }^{1}$, and Qin Shi ${ }^{1}$ \\ ${ }^{1}$ School of Mechanical Engineering, NJUST, Nanjing 210094, China \\ ${ }^{2}$ The $13^{\text {th }}$ Research Institute, CETC, Shijiazhuang 050051, China
}

\begin{abstract}
The dual-mass Silicon micro-machined gyroscope was processed by micro-fabrication technology. It could lead to quadrature coupling error and influence the output of the silicon micro-gyroscope. We select two commonly used gyroscope structures and analyze their quadrature coupling coefficient. Firstly, the complete dynamic model is proposed for the DMSG and the theoretical models of sensitivity and orthogonal signals are given by the dynamic model. Second, the influence of support structure on sensitivity and orthogonal signals are analyzed. The sensitivity and orthogonal signal of the two types of DMSG are derived and compared. The results show that the theoretical accuracy of the sensitivity and orthogonal signals can be improved about 50\% and $30 \%$ after considering the support structure. The type-B gyroscope are insensitive to the Coriolis force $(\approx 13 \%$ reduction) when compared to TypeA gyroscope. On the other hand the type-B gyroscope are insensitive to coupling stiffness ( $\approx 85 \%$ reduction) when compared to Type-A gyroscope. At last, the reliability of the theory is verified by simulations and experiments.
\end{abstract}

Keywords: Dual-mass micro-machined gyroscope, Dynamic model, Orthogonal signal, Sensitivity.

\section{Introduction}

The Dual-mass Silicon Micro-machined Gyroscopes (DSMG) have a lot of merits, such as low power consumption, small volume and weight, high shock resistivity, and suitable for the harsh conditions. They have a wide application prospect in civilian and military fields. It is a hot spot in the research of inertial technology.

Generally, the drive and sense modes of a Dual-mass Silicon Micro-machined Gyroscopes are designed to be perfectly orthogonal to each other. Limited by the current MEMS fabrication technology, the drive motion is coupled to the detection direction due to the coupled stiffness. The orthogonal signal is proportional to the drive-mode displacement. The phase of orthogonal signal is $90^{\circ}$ different from the useful signal so that can be rejected by using a phase-sensitive demodulation. However the detection signal of the silicon microgyroscope is very weak relative to the driver signal. Even very small phase errors still result

\footnotetext{
*Corresponding author: wangzhanghui19890917@njust.edu.cn
} 
in intolerable offset variations at the output of a gyroscope. Therefore, it is important to analyze the orthogonal signal of the DMSG.

The orthogonal signal of the DSMG had been analyzed in some literatures. The research had shown that the anisoelasticity was the main reason for the main source of the orthogonal signal. However the researches focus on the orthogonal signal of single-mass silicon micro-gyroscope. And the orthogonal signal of single-mass silicon micro-gyroscope is used to replace the orthogonal signal of the dual-mass silicon micro-machined gyroscope. With the improvement of the precision of the gyroscope, the influence of support structure on orthogonal signal cannot be ignored.

In this paper, two types of DSMG developed by our research group are taken as the research object. Firstly, the complete dynamic model is proposed for the DMSG, and the theoretical models of sensitivity and orthogonal signals are given by the dynamic model. Second, the influence of support structure on sensitivity and orthogonal signals are analyzed. The sensitivity and orthogonal signal of the two types of DMSG are derived and compared. At last, the reliability of the theory is verified by finite element method (FEM) dynamic simulation and experiments.

\section{Structure and operation principle}

Figure. 1 is the schematic of the dual-mass silicon micro-machined gyroscope. By applying the alternating voltage on the driving comb capacitor, the left and right mass will move in opposite direction along $\mathrm{X}$-axis (driving direction). When the sensor rotates about $\mathrm{Z}$-axis, the resulting Coriolis force causes the structure to move in opposite direction along Y-axis (sensing direction). The relative motion between the movable detection comb and the fixed detection comb forms the differential capacitance for detection. Ideally, the amount of differential detection capacitance is proportional to the input angular rate. We select two commonly used gyroscope structures to understand and to contrast their sensitivity to the coupling stiffness. The structures are shown in Figure 2. The detection comb of Type-A gyroscope is arranged on the proof mass, while the detection comb of Type-B gyroscope is arranged on the support structure.

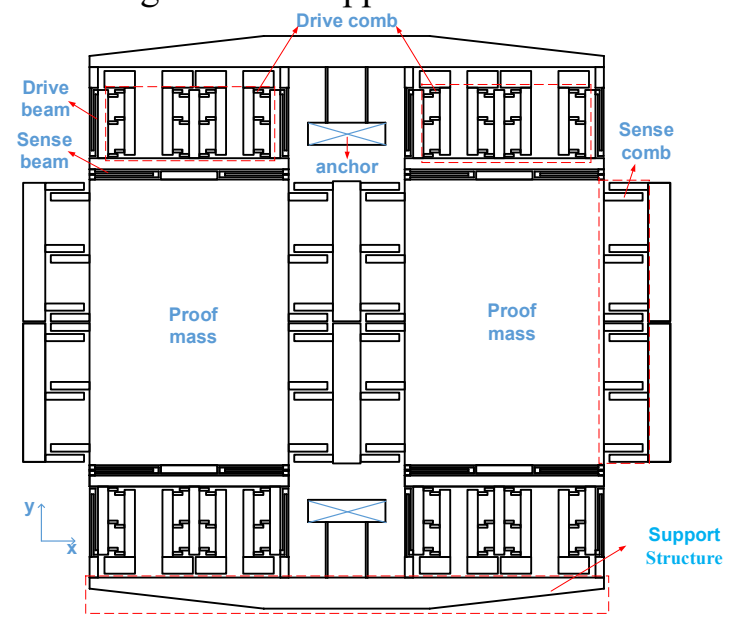

Fig. 1. The schematic of a dual-mass silicon micro-machined gyroscope. 


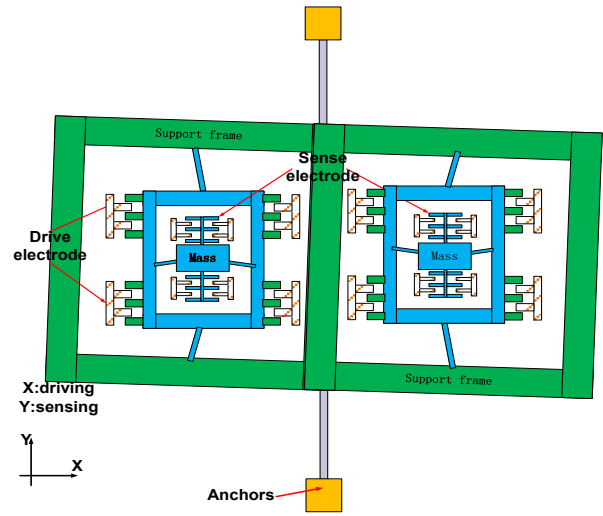

(a)

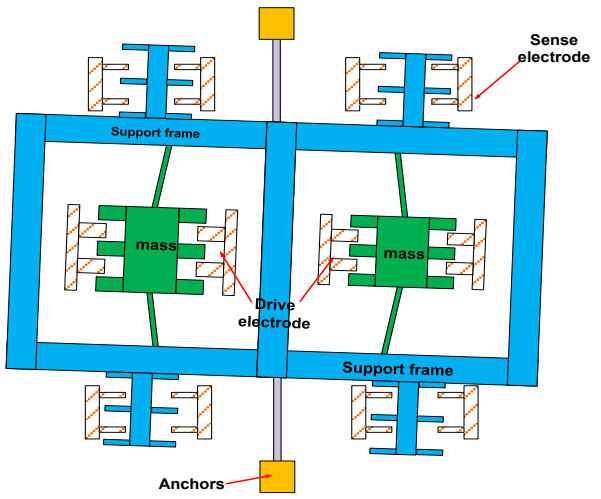

(b)

Fig. 2. Conceptual designs of two types of DSMG.

\subsection{Modeling \& discussion}

Figure 3. shows the dynamic model in the sensing direction. The motion equations are listed as below.

$$
\begin{gathered}
m_{d l} \ddot{y}_{L}+k_{d y l}\left(y_{L}+\theta l\right)=F_{L} \\
m_{d r} \ddot{y}_{R}+k_{d y r}\left(y_{R}-\theta l\right)=F_{R} \\
I_{b} \ddot{\theta}+k_{b} \theta+k_{d y}\left(y_{L}+\theta l\right) l-k_{d y}\left(y_{R}-\theta l\right) l=F_{b}
\end{gathered}
$$

Herein, $m_{d l}$ and $m_{d r}$ are the left and right proof masses, $I_{b}$ is the moment of inertia of the support frame, $k_{d y l}$ and $k_{d y r}$ are the stiffness in Y direction of the left and right drive beams, $F_{L}, F_{R}, F_{b}$ are the excitation forces applied to the left and right mass blocks and the supporting beams, respectively. $k_{b}$ is the stiffness of the supporting beam.

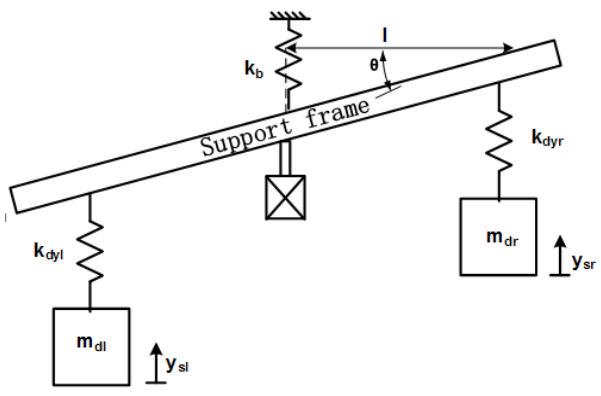

Fig. 3. The dynamic model in the sensing direction.

We obtain the motion equation for the mass-spring system expressed in a matrix form.

$$
[M]\{\vec{u}\}^{\prime \prime}+[k]\{\vec{u}\}=\{\vec{F}\} \sin \left(\omega_{d} t\right)
$$

where $[\mathrm{M}]$ and $[\mathrm{k}]$ are the mass and stiffness matrices respectively. These matrices and column vectors can be written as

$$
[M]=\left[\begin{array}{ccc}
m_{d l} & 0 & 0 \\
0 & m_{d r} & 0 \\
0 & 0 & I_{b}
\end{array}\right],[k]=\left[\begin{array}{ccc}
k_{d y l} & 0 & k_{d x l} l \\
0 & k_{d y r} & -k_{d y r} l \\
k_{d y l} l & -k_{d y r} l & k_{b}+k_{d y l} l^{2}+k_{d y r} l^{2}
\end{array}\right]
$$




\subsection{Sensitivity}

When the $Z$ axis has an angular velocity $\Omega_{Z}$ input, the masses are affected by the Coriolis force. Then the column vectors of the excitation force and the system displacement can be written as

$$
\left\{\vec{F}_{s}\right\}=\left[\begin{array}{lll}
F_{s}+\Delta F_{s} & F_{s}-\Delta F_{s} & 0
\end{array}\right]^{-1},\left\{\vec{u}_{s}\right\}=\left[\begin{array}{lll}
y_{s L} & y_{s R} & \theta_{s}
\end{array}\right]^{-1}
$$

where $F_{s}=2 M_{d} \Omega_{Z} \sum x$ and $\Delta F_{s}=2 M_{d} \Omega_{Z} \Delta x$ are the Coriolis force caused by translation motion and differential motion respectively. By defining $\left.\left[z\left(\omega_{d}\right)\right]=\left[k-m \omega_{d}^{2}\right)\right]$, the equations can be written as below.

$$
\left[z\left(\omega_{d}\right)\right]\left\{\vec{u}_{s}\right\}=\left\{\vec{F}_{s}\right\}
$$

The response of the system can be obtained by the inverse matrix method.

$$
\left\{\vec{u}_{s}\right\}=\left[z\left(\omega_{d}\right)\right]^{-1}\left\{\vec{F}_{s}\right\}
$$

Assume $\mathrm{m}_{\mathrm{dl}}=\mathrm{m}_{\mathrm{dr}}=\mathrm{m}_{\mathrm{d}}, \mathrm{k}_{\mathrm{dyl}}=\mathrm{k}_{\mathrm{dyr}}=\mathrm{k}_{\mathrm{dy}}$.

$$
\begin{gathered}
{\left[z\left(\omega_{d}\right)\right]^{-1}=\left[\begin{array}{ccc}
z_{d}\left(\omega_{d}\right) z_{b}\left(\omega_{d}\right)-k_{d y}^{2} l^{2} & k_{d y}^{2} l^{2} & -z_{d}\left(\omega_{d}\right) k_{d y} l \\
k_{d y}^{2} l^{2} & z_{d}\left(\omega_{d}\right) z_{b}\left(\omega_{d}\right)-k_{d y}^{2} l^{2} & z_{d}\left(\omega_{d}\right) k_{d y} l \\
-z_{d}\left(\omega_{d}\right) k_{d y} l & z_{d}\left(\omega_{d}\right) k_{d y} l & z_{d}^{2}\left(\omega_{d}\right)
\end{array}\right]\left[\left(z_{d}^{2}\left(\omega_{d}\right) z_{b}\left(\omega_{d}\right)-2 z_{d}\left(\omega_{d}\right) k_{d y}^{2} l^{2}\right)\right]^{-1}} \\
z_{d}\left(\omega_{d}\right)=k_{d y}-m_{d} \omega_{d}^{2}, z_{b}\left(\omega_{d}\right)=k_{b}+2 k_{d y} l^{2}-I_{b} \omega_{d}^{2}
\end{gathered}
$$

Substituting equation (4) and (7) into (6), we can obtain the solution of equation (2).

$$
\begin{gathered}
y_{s L}=\left\{\left[z_{d}\left(\omega_{d}\right) z_{b}\left(\omega_{d}\right)-2 k_{d y}^{2} l^{2}\right] F_{s}+z_{d}\left(\omega_{d}\right) z_{b}\left(\omega_{d}\right) \Delta F_{s}\right\}\left[z_{d}^{2}\left(\omega_{d}\right) z_{b}\left(\omega_{d}\right)-2 z_{d}\left(\omega_{d}\right) k_{d y}^{2} l^{2}\right]^{-1} \\
y_{s R}=\left\{\left[z_{d}\left(\omega_{d}\right) z_{b}\left(\omega_{d}\right)-2 k_{d y}^{2} l^{2}\right] F_{s}-z_{d}\left(\omega_{d}\right) z_{b}\left(\omega_{d}\right) \Delta F_{s}\right\}\left[z_{d}^{2}\left(\omega_{d}\right) z_{b}\left(\omega_{d}\right)-2 z_{d}\left(\omega_{d}\right) k_{d y}^{2} l^{2}\right]^{-1} \\
\theta_{s}=-2 z_{d}\left(\omega_{d}\right) k_{d y} l \Delta F_{s}\left(z_{d}^{2}\left(\omega_{d}\right) z_{b}\left(\omega_{d}\right)-2 z_{d}\left(\omega_{d}\right) k_{d y}^{2} l^{2}\right)^{-1} .
\end{gathered}
$$

The detection displacement that caused by the Coriolis force are shown as

$$
\begin{array}{r}
y_{s A}=\left(y_{s L}-y_{s R}\right) / 2=z_{b}\left(\omega_{d}\right) \Delta F_{s}\left[I_{b} m_{d}\left(\omega_{s}^{2}-\omega_{d}^{2}\right)\left(\omega_{g}^{2}-\omega_{d}^{2}\right)\right]^{-1} \\
y_{s B}=\theta_{s} l=-2 k_{d y} l^{2} \Delta F_{s}\left[I_{b} m_{d}\left(\omega_{s}^{2}-\omega_{d}^{2}\right)\left(\omega_{g}^{2}-\omega_{d}^{2}\right)\right]^{-1}
\end{array}
$$

Herein, $w_{s}$ and $w_{g}$ are the natural frequencies of the detection mode and the high order mode. The value are given by

$$
\omega_{(s, g)}^{2}=\left\{\left(m_{d} k_{b}+2 m_{d} k_{d y} l^{2}+k_{d y} I_{b}\right) \mp \sqrt{\left(m_{d} k_{b}+2 m_{d} k_{d y} l^{2}+k_{d y} I_{b}\right)^{2}-4 k_{d y} k_{b}}\right\}\left(2 I_{b} m_{d}\right)^{-1}
$$

The sensitivity of Type-A and Type-B gyroscopes are given by

$$
S F_{A}=y_{s A} / \Omega_{z}, S F_{B}=y_{s B} / \Omega_{z}
$$

According to equation (11)-(15), the relationship of sensitivity between the two types of DSMG is given by

$$
S F_{B} / S F_{A}=2 k_{d y} l^{2}\left(k_{b}+2 k_{d y} l^{2}-I_{b} \omega_{d}^{2}\right)^{-1}
$$

Obviously, the relation in equation (15) means the sensitivity of the two gyroscopes depends on the driving frequency of the system, the stiffness of the supporting beam, the sensitive mass and the moment of inertia of the support structure. Due to the difference of gyro parameters, the sensitivity can be increased by selecting different detection methods (Type-A or Type-B). 


\subsection{Orthogonal Signal}

Limited by the current MEMS fabrication technology, the driven displacement is coupled to the detection direction by coupling stiffness. Then the column vectors of the excitation force and the system displacement can be written as

$$
\left\{\vec{F}_{o}\right\}=\left[\begin{array}{lll}
F_{o}+\Delta F_{o} & F_{o}-\Delta F_{o} & 2 \Delta F_{o} l
\end{array}\right]^{-1},\left\{\begin{array}{l}
\vec{u}_{o} \\
y
\end{array}=\left[\begin{array}{lll}
y_{o L} & y_{o R} & \theta_{o}
\end{array}\right]^{-1}\right.
$$

where $F_{o}=\sum \varepsilon k_{d y} x$ and $\Delta F_{o}=\Delta \varepsilon k_{d y} x$ are the coupling force caused by the stiffness coupling coefficient. The orthogonal displacement caused by the stiffness coupling coefficient can be obtained by the inverse matrix method.

$$
\begin{gathered}
y_{o A}=\left[z_{b}\left(\omega_{d}\right)-2 k_{d y} l^{2}\right] \Delta F_{o}\left[I_{b} m_{d}\left(\omega_{s}^{2}-\omega_{d}^{2}\right)\left(\omega_{g}^{2}-\omega_{d}^{2}\right)\right]^{-1} \\
y_{o B}=2 m_{d} \omega_{d}^{2} \Delta F_{o} l^{2}\left[I_{b} m_{d}\left(\omega_{s}^{2}-\omega_{d}^{2}\right)\left(\omega_{g}^{2}-\omega_{d}^{2}\right)\right]^{-1}
\end{gathered}
$$

According to the equation (14), (17), (18), the orthogonal signal of the two types of DSMG are given by

$$
\alpha_{A}=y_{o A} / S F_{A}, \alpha_{B}=y_{o B} / S F_{B}
$$

According to the equation (19), the relationship of orthogonal signal caused by the coupling stiffness between the two types of DSMG is given by

$$
\alpha_{B} / \alpha_{A}=\left(k_{b}-I_{b} \omega_{d}^{2}\right) k_{d y}\left[\left(k_{b}+2 k_{d y} l^{2}-I_{b} \omega_{d}^{2}\right) m_{d} \omega_{d}^{2}\right]^{-1}
$$

\subsection{Discussions}

The equation (11), (12), (17), (18) shows that the sensitivity and orthogonal signal are related to the support system. The parameters of the DMSG designed are listed in the Table 1. And the stiffness coupling coefficient due to the process error is about 5e-5. Figure 4 is the relationship between the moment of inertia of the support structure and the sensitivity. Figure 5 is the relationship between the moment of inertia of the support structure and the orthogonal signal.

Table 1. The parameters of the DMSG designed.

\begin{tabular}{ccccccc}
\hline Parameter Name & $M_{b}$ & $I_{b}$ & $k_{b}$ & $l$ & $w d$ & $k_{d y}$ \\
\hline Value & 1.89 & $1.98 e-13$ & 0.0115 & 811 & 18911 & 50000 \\
Unit & $e-7 k g$ & $\mathrm{~kg}^{*} \mathrm{~m}^{\wedge} 2$ & $\mathrm{~N} / \mathrm{m}$ & $\mathrm{um}$ & $\mathrm{Hz}$ & $\mathrm{N} / \mathrm{m}$ \\
\hline
\end{tabular}




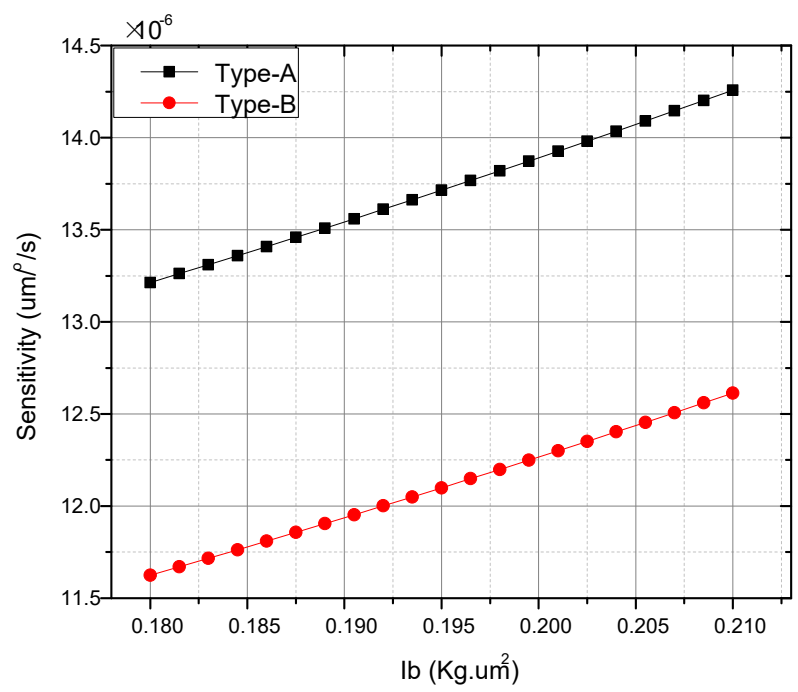

Fig. 4. The relationship between the moment of inertia of the support structure and the sensitive signal.

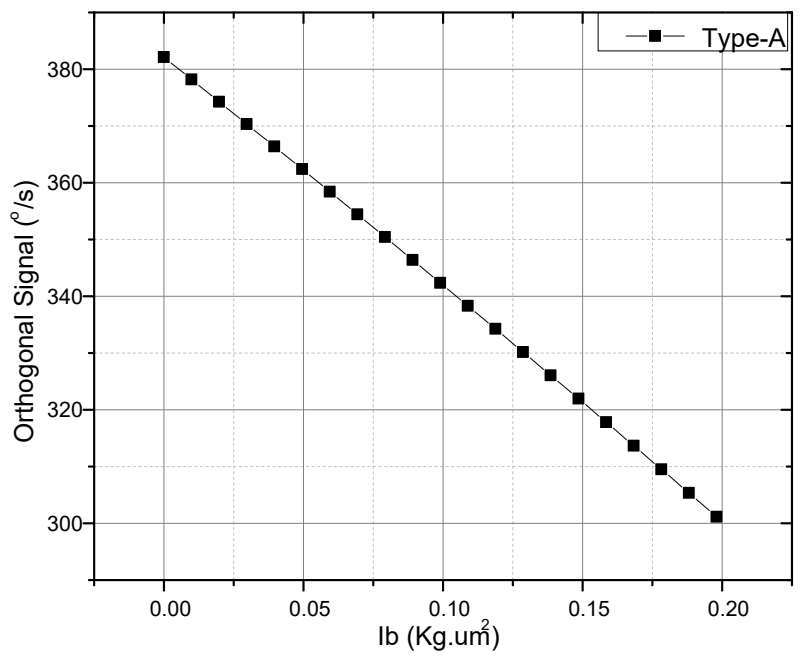

Fig. 5. The relationship between the moment of inertia of the support structure and the orthogonal signal.

Figure 4 shows that the sensitivity can be increased by the increasing the moment of inertia of the support structure. By considering the support structure, the sensitive signal is about $50 \%$ different from the model without the support structure. Figure. 5 shows that the orthogonal signal can be reduced by the increasing the moment of inertia of the support structure. By considering the support structure, the orthogonal signal is about $30 \%$ different from the model without the support structure.

According to equation (15), the Type-B gyroscope are insensitive to the Coriolis force $(\approx 13 \%$ reduction) when compared to Type-A gyroscope. As a result, Type-A gyroscope is selected to increase the sensitivity of DSMG.

According to equation (18), the type-B gyroscope are insensitive to coupling stiffness ( $\approx 84 \%$ reduction) when compared to Type-A gyroscope. As a result, Type-B gyroscope is selected to reducing the orthogonal signal and improving the performance of DSMG. 


\section{Fem simulation}

The Finite element software ANSYS was employed to verify the theoretical model. The schematic of a dual mass silicon micro-machined gyroscope is shown in Figure 6.

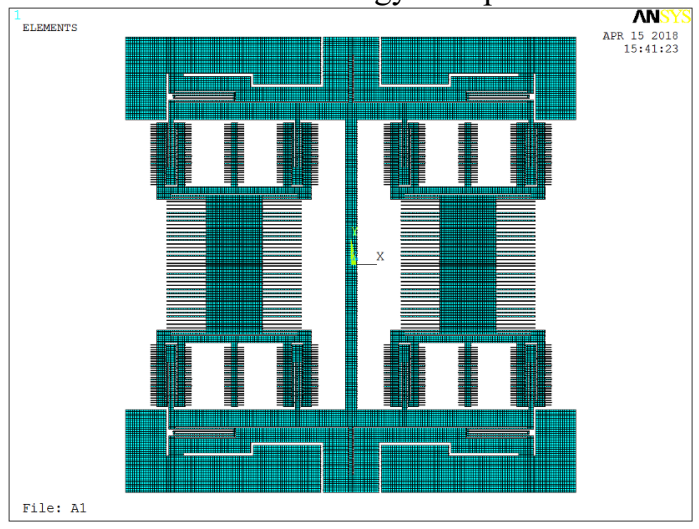

Fig. 6. The schematic of a dual mass silicon micro-machined gyroscope.

Because of the high natural frequency, the harmonic response analysis is used instead of the transient analysis to simulate the dynamic response of the dual mass silicon micromachined gyroscope.

\subsection{Sensitivity simulation}

The anchor are restrained, and the equivalent Coriolis force of $1 \mathrm{e}-6 \mathrm{~N}$ is applied to all nodes of the left and right mass respectively. The sensitivity is reflected by the radio of $\mathrm{Y}$ direction displacement and the equivalent angular rate. The frequency range of $18 \mathrm{KHz}$ to $20 \mathrm{KHz}$ (include the natural frequency in its driving direction). The number of steps is 100 . Figure 7 shows the sensitivity of theory and simulation of the two types of gyroscopes. According to Figure 7 the sensitivity is about $1 \mathrm{e}-5 \mathrm{um} / \%$ s. And the Type-B gyroscope are insensitive to the Coriolis force when compared to Type-A gyroscope. It also shows that the theoretical result and simulation result are in good agreement (theoretical value is $8.3 \%$ different from the simulation value).

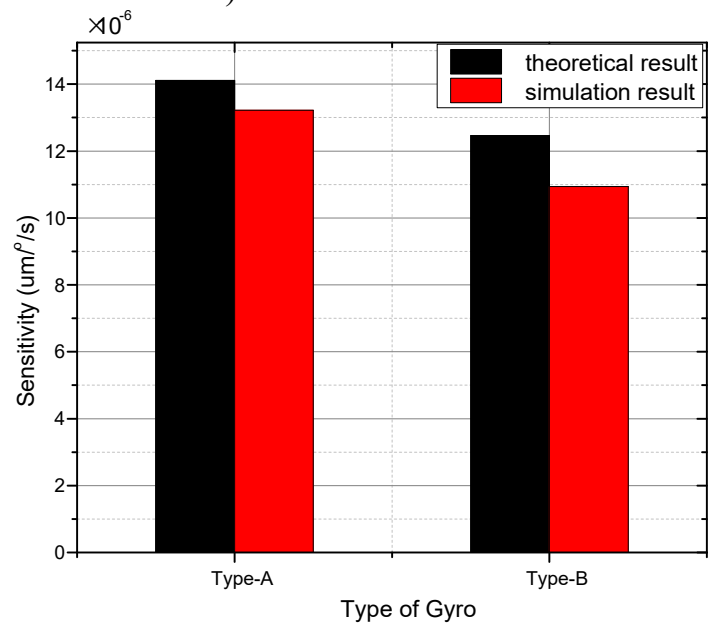

Figure 7. The sensitivity of theory and simulation of two types of gyroscopes. 


\subsection{Orthogonal signal simulation}

The driving force of $1 e-6 \mathrm{~N}$ is applied to all nodes of the left and right mass respectively. The orthogonal signal caused by the coupling stiffness is reflected by the radio of $\mathrm{Y}$ direction displacement and sensitivity. The frequency range of $18 \mathrm{KHz}$ to $20 \mathrm{KHz}$ (include the natural frequency in its driving direction). The number of steps is 100 . Figure 8 shows the orthogonal signal of theory and simulation of the two types of gyroscopes. According to Figure 8 the orthogonal signals of the two types of gyroscopes are about $300^{\circ} / \mathrm{s}$ and $60^{\circ} / \mathrm{s}$ respectively. And the Type-B gyroscope are insensitive to coupling stiffness $(\approx 84 \%$ reduction) when compared to Type-A gyroscope. It also shows that the theoretical result and simulation result are in good agreement.

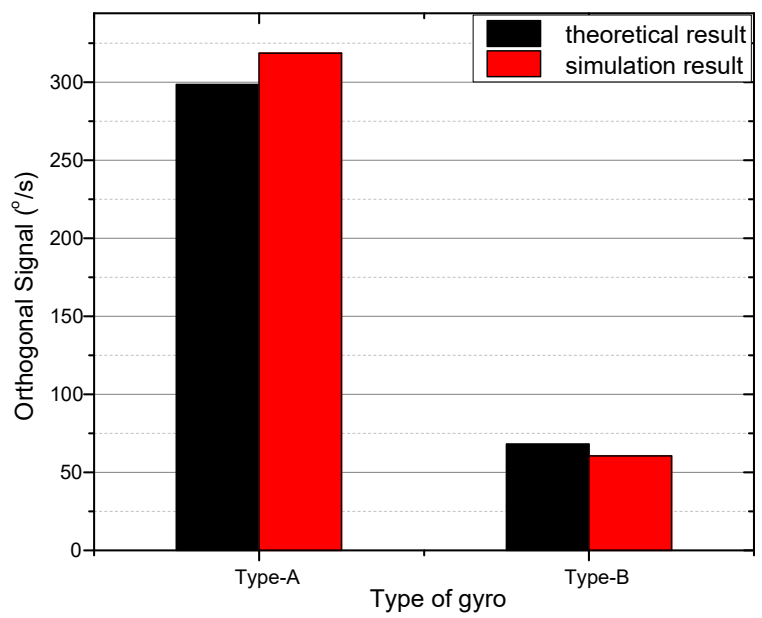

Fig. 8. The orthogonal signal of theory and simulation of two types of gyroscopes.

\section{Experiment}

In this paper, the orthogonal signals of two types of gyroscope are tested respectively. The $\mathrm{AC}$ voltage with DC bias is applied on the drive comb of the silicon micro gyroscope. The output voltage $U$ without angular rate input is mainly orthogonal signal. The orthogonal signals can be obtained by the ratio of output voltage and scale factor.

To achieve a fair comparison, the Type-A and Type-B gyroscopes are selected to have the similar parameters. The experimental results of the orthogonal signal of the two types of gyroscopes are shown in Table 3 and Table 4. The data shows that the orthogonal signal of the type-B gyroscope is smaller when compared to the Type-A gyroscope. Therefore, the Type-B gyroscope is selected to reducing the orthogonal signal and improving the performance of DSMG.

Table 3. The experimental results of orthogonal signal of the Type-A gyroscopes.

\begin{tabular}{cccc}
\hline & scale factor $(m v / \% / s)$ & output voltage $(m v)$ & orthogonal signal $(\% / s)$ \\
\hline 1 & 0.61 & 165 & 270 \\
2 & 2.22 & 709 & 297 \\
3 & 4.34 & 823 & 174 \\
\hline
\end{tabular}

Table 4. The experimental results of orthogonal signal of the Type-B gyroscopes.

\begin{tabular}{cccc}
\hline & scale factor $(\mathrm{mv} / \mathrm{o} / \mathrm{s})$ & output voltage $(\mathrm{mv})$ & orthogonal signal $\left({ }^{\circ} / \mathrm{s}\right)$ \\
\hline 1 & 0.55 & 62 & 105
\end{tabular}




\begin{tabular}{lccc}
2 & 1.03 & 81 & 73 \\
3 & 4.12 & 281 & 64 \\
\hline
\end{tabular}

\section{Conclusion}

Base on the structural characteristics of the two types of gyroscopes, the complete dynamic model is proposed for the DMSG and the theoretical models of sensitivity and orthogonal signals are given by the dynamic model. The results show that the sensitivity is about $1 \mathrm{e}-$ $5 \mathrm{um} /{ }^{\circ} / \mathrm{s}$ and the orthogonal signals of the two types of gyroscopes are about $300 \% \mathrm{~s}$ and $60 \%$ respectively. The results also shows that the type-B gyroscope are insensitive to the Coriolis force ( $\approx 13 \%$ reduction) when compared to Type-A gyroscope. On the other hand the Type-B gyroscope are insensitive to coupling stiffness ( $\approx 85 \%$ reduction) when compared to Type-A gyroscope. At last, the reliability of the theory is verified by simulations and experiments. The good agreement between the theory result and simulation result prove the accuracy of our model and its theory.

\section{References}

1. M. S. Weinberg and A. Kourepenis, "Error sources in in-plane silicon tuning-fork MEMS gyroscopes," J. Microelectromechanical. Syst., vol. 15, no.3, pp. 479-491, Jun. 2006.

2. SH D. Jiang, A P. Qiu, Q. Shi, “Calculation and verification of quadrature coupling coefficient of silicon micro-gyroscope,” [J]. Opt. Precision Eng. , 2013, 21(1): 87-93.

3. B. Yang, B L. Zhou. "Analysis of Quadrature Error of Dual - Frame Silicon Micromachined Gyroscope," International Conference on Electrical and Control Engineering. Sept. 16-18, 2011. pp. 3931-3934

4. B. Yang, X. Wang, D. Hu, and L. Wu, "Research on the non-ideal dynamics of a dualmass silicon micro-gyroscope," Microsystem Technologies, vol. 23, no. 1, pp. 151-162, 2017

5. E. Tatar, S. E. Alper, and T. Akin, "Effect of quadrature error on the performance of a fully-decoupled MEMS gyroscope," in Proc. Int. Conf. MEMS, Cancun, Mexico, Jan. 2011, pp. 569-572

6. B. Yang, X. Wang, Y. Deng, et al. Mechanical Coupling Error Suppression Technology for an Improved Decoupled Dual-Mass Micro-Gyroscope[J]. Sensors, 2016, 16(4):503. 